\title{
Umbilical Pilonidal Sinus: A Case Report
}

\author{
Nezih Akkapulu ${ }^{\mathrm{a}, \mathrm{b}}$, Yusuf Tanrikulu ${ }^{\mathrm{a}}$
}

\begin{abstract}
Pilonidal sinus disease is a recurrent and chronic disorder that can be observed in the periumbilical area although commonly seen in sacrococygeal region. Sinus excision with reconstruction of the umbilicus is a surgical option for treatment of umbilical pilonidal sinus disease. We report a case of 21-year-old male patient with umbilical pilonidal sinus that was treated by sinus excision with reconstruction of the umbilicus, which is an agreeable and alternative treatment option of umbilectomy.
\end{abstract}

Keywords: Umbilical pilonidal sinus; Sinus excision; Umbilicus reconstruction

\section{Introduction}

Pilonidal sinus is a disease which is characterized by a granulomatous reaction to a hair shaft penetrating epidermis from the cutaneous surface [1]. Although commonly seen in sacrococygeal region, it is also observed in the periumbilical area [2]. Umbilical pilonidal sinus is a quite uncommon disease. Even though there is no particular agreement about the treatment of this disease, various and different treatment methods (from conservative modalities to umbilectomy) exist in literature [1, 3-8]. We present a patient with umbilical pilonidal sinus who was successfully treated by sinus exci-

Manuscript accepted for publication October 5, 2011

${ }^{a}$ Erzincan Military Hospital, Department of General Surgery, 24000, Erzincan, Turkey

bCorresponding author: Nezih Akkapulu, Email: akkapulu@gmail.com

doi:10.4021/jmc348w sion with conserving umbilicus.

\section{Case Report}

Twenty-one-year-old Turkish male patient presented with a four-year history of intermittent, recurrent bloody discharge, umbilical pain and swelling of umbilicus. Medical history was unremarkable. On examination, it was observed that he had a hairy body, there was a serohemorrhagic discharge from inside the umbilicus and there were unbound shafts. Laboratory tests did not yield to any particular finding. No problems were observed when superficial and intraabdominal ultrasound imaging was performed.

Umbilectomy was recommended to the patient however he did not accept because he did not want his navel to be removed. Performing sinus excision with umbilicus reconstruction was decided with informed consent.

Prophylaxis was performed via intravenous route with one g. Cefazolin Sodium and then under general anesthesia, a curved incision was made along the inferior border of the umbilicus from three o'clock to nine o'clock. The incision was deepened to the fascia along the full length of the incision. The umbilicus was completely everted after it was dissected and detached from the fascia. The sinus which was presented along subcutaneously was seen (Fig. 1) and it was excised by wedge fashion. Preformed defect was repaired

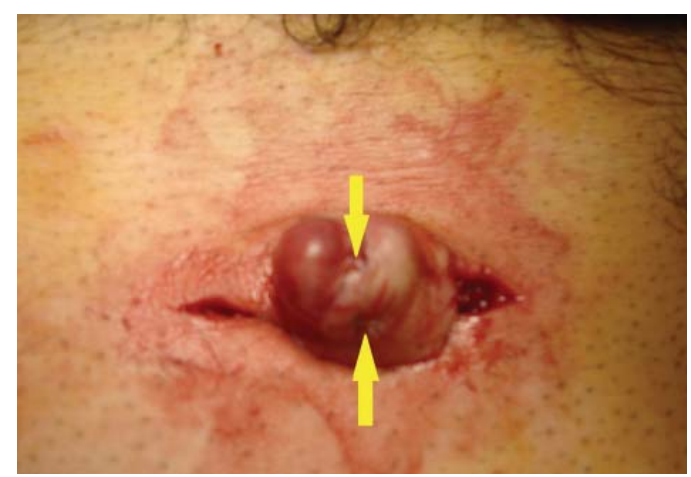

Figure 1. The sinus which was presented along subcutaneously was seen and it was excised by wedge fashion. 


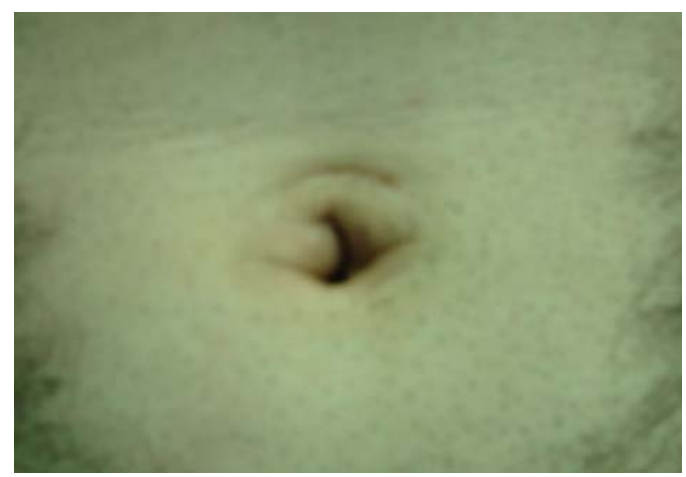

Figure 2. Pre-operative appearance of the umbilicus

by separate absorbable sutures from underneath. The center of new umbilicus was secured to fascia by single delayed absorbable suture. Then the skin was closed by running subcuticular fashion with non-absorbable suture.

No antibiotic was administered to the patient in postoperative period. The suture was removed at seventh postoperative day. Histopathologic evaluation was compatible with pilonidal sinus disease. Follow-up examinations were performed at the first, third and sixth months after the surgery, and no recurrence was observed throughout in this period. The patient found the appearance of the new umbilicus "acceptable” (Fig. 2, 3).

\section{Discussion}

Umbilical pilonidal sinus was first described 160 years ago [9]; however there were less than 60 patients that were reported by surgical treatment in international (English-written) literature.

Congenital origin theory is losing popularity against acquired origin theory of pilonidal sinus disease [10]. Eryilmaz et al. [1] described predisposing factors for umbilical pilonidal sinus such as hairiness, male sex, young age, and poor personal hygiene. Moreover, the same study listed the most common symptoms as umbilical pain, bloody discharge, purulent discharge and umbilical mass with 100\%, 69\%, 23\% and $26 \%$ respectively [1].

Other umbilical region anomalies such as umbilical hernia, endometriosis (for women), Sister Mary Joseph nodule, pyogenic granuloma, urachus and epidermoid cysts should be kept in mind at the differential diagnosis. A pre-operative intra-abdominal imaging may be beneficial $[6,11]$.

There is not any consensus about the treatment of umbilical pilonidal sinus disease, however, failures of conservative methods such as hair extraction and appropriate personal hygiene and recurrent cases make the surgical treatment an inevitable option [1, 3-5]. Umbilectomy is advised for cessation recurrence by some authors $[1,7]$. However, no re-

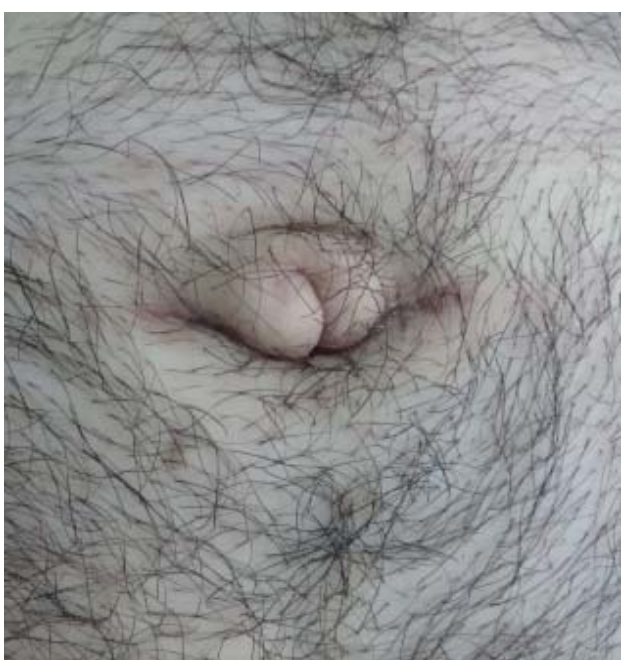

Figure 3. The patient found the appearance of the new umbilicus "acceptable"

currence survey was shown in the cases with sinus excision and preserving umbilicus $[5,8]$. Presenting case suffers from short survey interval like as 6 months, but recurrence was not shown in the 45 cases of sinus excision with umbilicus reconstruction [5]. This method is a more feasible option than umbilectomy cosmetically.

In conclusion sinus excision with umbilicus reconstruction is a treatment option of the umbilical sinus disease which is easily performed, yields acceptable results cosmetically and is alternative option for the umbilectomy.

\section{Conflict of Interest}

Nezih Akkapulu M.D. and Yusuf Tanrikulu M.D. have no conflict of interest.

\section{References}

1. Eryilmaz R, Sahin M, Okan I, Alimoglu O, Somay A. Umbilical pilonidal sinus disease: predisposing factors and treatment. World J Surg. 2005;29(9):1158-1160.

2. Hull TL, Wu J. Pilonidal disease. Surg Clin North Am. 2002;82(6):1169-1185.

3. Abdelnour A, Aftimos G, Elmasri H. Conservative surgical treatment of 27 cases of umbilical pilonidal sinus. J Med Liban. 1994;42(3):123-125.

4. El-Bakry AA. Discharging umbilicus. Saudi Med J. 2002;23(9):1099-1100.

5. Fazeli MS, Lebaschi AH, Adel MG, Kazemeini AR. Evaluation of the outcome of complete sinus excision with reconstruction of the umbilicus in patients with umbilical pilonidal sinus. World J Surg. 2008;32(10):2305- 
2308.

6. Abdulwahab BA, Harste K. Umbilical pilonidal sinus. Ugeskr Laeger. 2010;172(41):2848-2849.

7. Sroujieh AS, Dawoud A. Umbilical sepsis. Br J Surg. 1989;76(7):687-688.

8. Naraynsingh V, Hariharan S, Dan D. Umbilical pilonidal sinus: a new treatment technique of sinus excision with umbilical preservation. Dermatol Surg.
2009;35(7):1155-1156.

9. Thorlakson RH. Pilonidal sinus of the umbilicus. Br J Surg. 1966;53(1):76-78.

10. Humphries AE, Duncan JE. Evaluation and management of pilonidal disease. Surg Clin North Am. 2010;90(1):113-124, Table of Contents.

11. McClenathan JH. Umbilical pilonidal sinus. Can J Surg. 2000;43(3):225. 